\title{
GESNERUS
}

Vierteljahresschrift für Geschichte der Medizin und der

Naturwissenschaften

Revue trimestrielle d'histoire de la médecine

Jahrgang - 5 - Volume $1948 \quad$ Heft - Fasc. $-3 / 4$

Der Stadtarzt Dr. Sebastian Sdobinger in St. Gallen

(1579-1652)

Von P. Jung, St. Gallen

Unsere schweizerische Gesellschaft für Geschichte der Medizin und der Naturwissenschaften hat zu wiederholten Malen lebhaftes Interesse bekundet für Hinweise auf sonst leider nur wenig bekannte und doch in ihrer Zeit überragende Gestalten der Medizin und Naturwissenschaft. Wer sich ihnen nähert, wird immer bedauern, daß sich nicht Historiker vom Fach der Aufgabe ausschließlich widmen. Gerade unsere St. Galler Bibliotheken bieten dafür ein reiches Material. Für heute greife ich eine Persönlichkeit heraus, die vermöge der glänzenden Gaben und ihres umfassenden Wissens zu den Führergestalten ihrer Zeit in engster Beziehung stand und von diesen hochgeschätzt wurde.

Sebastian Schobinger, geb. 10. April 1579, gest. 10. Januar 1652, hat am 7. September 1601 in Basel mit der These "de Paralysi» dissertiert. Die übliche Disputation behandelt zeitentsprechend metaphysische Probleme, u. a. "An alliquid nobis a natura sit cognitum?" - "An Dificultas intelligendi dependeat a rebus intelligendis an potius ab ipso intellectu? » Themen, die auch heute im Zeitalter der Existentialphilosophie an Aktualität nichts eingebüßt haben.

Schobinger wird als Arzt, Gelehrter und Staatsmann bezeichnet. 1611 wurde er mit 32 Jahren Stadtarzt; er galt als der «bedeutendste Arzt der Ostschweiz» und war als praktischer Arzt wissenschaftlich und politisch tätig. Er war fürstlich-sanktgallischer Stiftsarzt, Ratsherr, Bibliothekar der Vadiana, von 1618 bis 1629 Tagsatzungsgesandter, zwanzig Jahre lang (1632-1652) Bürgermeister und wurde am 13. März 1623 in den Reichsadelsstand erhoben mit acht Brüdern und allen Nachkommen seines Großvaters Bartholomäus. 
Die Schobinger waren seit alters ein naturwissenschaftlich vielseitig interessiertes Geschlecht. Als erster seines Namens wird Hans Schobinger als Bürger von St. Gallen genannt; dessen Sohn Bartholome (1500-1585), der Großvater Sebastians, trug den Beinamen der «reiche Philosoph». Als Eisenhändler im Hause Notveststein (die heutige Wegelinbank) war er zu großem Besitz gelangt. Von 1550 bis 1582 bekleidete er die Würde eines Ratsherrn; er galt als Förderer der Reformation und als Gelehrter. Das Studium hatte er wegen "schwerer Zunge» aufgeben müssen.

Seine Studien galten der Alchemie, "worüber er auf seinem Schloß Horn am Bodensee 1531 mit Paracelsus disputierte». So liest man es durchwegs in der einschlägigen Literatur. Die Angabe ist nachweisbar falsch. Eine Nachfrage beim thurgauischen Staatsarchiv ergibt nämlich folgendes: Ein Kaspar Schobinger schrieb 1661, die Schobinger seien jetzt 82 Jahre auf Haus und Gut zu Horn und es sei nicht recht, daß man sie von dort vertreibe. Vor 1579 waren die Schobinger nicht in Horn, nachweisbar erst 1586. Die Bezeichnung «Schloß» kommt erst 1682 vor. Früher ist nur von "Haus und Gut» die Rede. Es ist also urkundlich erwiesen, daß die Schobinger 1531, wie allgemein zu lesen ist, gar nicht in Horn waren, sondern erst 48 Jahre später. Da aber bekanntlich Paracelsus am 24. September 1541 in Salzburg starb und Horn damals noch gar nicht im Besitz der Schobinger war, so kann auch die fragliche Aussprache zwischen Bartholome, dem Großvater Sebastians, und Paracelsus in Horn 1531 gar nicht stattgefunden haben. Es handelt sich da, wie so oft, um eine Legende, die einer dem andern nachdruckt, ohne der Frage auf den Grund zu gehen.

Über seine Schriften gibt ein "Catalogus librorum Bartholomäi Schobingeri» der Vadiana Auskunft. Von Paracelsus besaß er ein Originalporträt, das von seinen Nachkommen testamentarisch als Bestandteil seiner Bibliothek jeweilen dem ältesten Studierenden der Familie zubestimmt wurde. Aber schon nach dem Tode seines Enkels Sebastian entstand unter den Erben ein Prozeß über den Besitz der Bücher und des Paracelsus-Bildes, der zwei Monate nach dem Tode Sebastians von Bürgermeister und Rat durch Spruch vom 4. März 1652 entschieden wurde. Die Kopie jenes Schiedsspruchs soll später aufgefunden worden sein, ließ sich aber seither nicht mehr feststellen. Das fragliche Paracelsus-Bild hängt heute in der Apotheke des Sanktgallischen Historischen Museums und trägt die Jahrzahl 1520. Von fachkundiger Seite (Frl. Dr. Rittmeyer) wird aber entschieden betont, daß es sich nicht um ein Original von 1520 handle, sondern um eine Kopie, vermutlich aus dem 17. Jahrhundert. 
Von den beiden Söhnen Bartholomes war Tobias als Mathematiker und Architekt angesehen; von David sind 33 Briefe erhalten. Ein Neffe Sebastians, Sohn seines Bruders Esaias, und sein Nachfolger als Stadtarzt 1635, war Heinrich Schobinger. Auf Briefe an seinen Onkel kommen wir zurück. Er war Famulus bei Fabricius Hildanus in Bern.

Der Freundlichkeit von Prof. Wegelin, Bern und St. Gallen, verdanke ich ferner die Kenntnis einer Basler Dissertation von 1637 eines Verwandten Seb. Schobingers, wieder eines Bartholome, der von 1610 bis 1675 lebte. An erster Stelle ist die Dissertation Seb. Schobinger gewidmet: "Rei publicae San Gallensis Consuli gravissimo». Ihr Thema "Lithologia medica" behandelt Pathologie und Therapie der Steinkrankheiten im allgemeinen und besonders der Blasensteine mit einem speziellen Hinweis auf die Lithotomie, wie sie von Fabricius Hildanus in Bern geübt wurde.

Da wir gerade bei der Genealogie sind, mag interessieren, daß Johann Jakob Wepfer am 11. Oktober 1678 an Johannes von Muralt nach Zürich schreibt von einer "gebildeten weiblichen Geburtshelferin Schobinger aus vornehmem Hause in St. Gallen. Die vortreffliche Dame Kleophea Schobingerin aus der adeligen Familie Zollikofer» sucht durch Wepfer eine Unterredung mit von Muralt nach. Anscheinend, soweit sich das verfolgen läßt, muß es sich um eine Großnichte Seb. Schobingers gehandelt haben. Ihre Geistesgaben werden in dem Empfehlungsschreiben als «unvergleichlich und über den Horizont ihres Geschlechtes hinausgehend» bezeichnet. Sie sei mehr als einmal Schwergebärenden beigestanden und soll nun die Extractio Foetuum erlernen. Zunächst zwar gegen ihren Willen, da sie vermöge ihres Reichtums zu Hause in beschaulicher Ruhe leben könnte. Sie läßt dann aber zu ihrer Belehrung Frauenleichen sezieren und sucht nun zur Vollendung ihrer Ausbildung, durch die Vermittlung Wepfers, von Muralt auf. Johann Conrad Peyer nennt sie in einem Briefe an von Muralt vom 7. April 1680 "eine göttliche und heilbringende Stütze der Schwergebärenden». Aus diesen genealogischen Andeutungen erhellt zur Genüge, daß es sich bei dem Geschlecht der Schobinger zweifellos um medizinisch und naturwissenschaftlich stark interessierte Persönlichkeiten handelte.

Nun zu Sebastian Schobinger selbst. - An Sebastian Schobinger sind in der Vadiana 701 Briefe erhalten, leider keine von ihm. Es sind beredte Zeugen von dem außergewöhnlichen Ansehen, das Seb. Schobinger als Arzt, Naturwissenschaftler und Politiker genossen haben muß. Ein großer Teil dieser Briefe stammen von seinem oben erwähnten Neffen und spä- 
teren Nachfolger als Stadtarzt Heinrich Schobinger. Je nach dem Standort schreibt er Französisch oder Deutsch. Er berichtet 1628 über den Studienbetrieb bei Fabricius und erzählt von Operationen, die er dort zu sehen Gelegenheit hatte. Von Basel aus bespricht er Fragen des medizinischen Unterrichts, teilt 1629 und 1631 aus Ems und Konstanz Krankengeschichten mit, berät ihn öfter wegen eines Patienten, "periculosissime» erkrankt. Dazwischen unterhält er sich mit seinem Onkel von Genf aus über politische und persönliche Angelegenheiten, bittet ein andermal um Empfehlungen an Konstanzer Notabilitäten oder um Zimmerbestellung im Bad Fideris für den Comte de Zylle. Es folgen Reiseberichte und Aussprachen über politische Tagesereignisse. Schobingers Autorität als Arzt künden Bitten von Kranken und deren Angehörigen um ärztlichen Rat, so vom Landammann und Rat in Appenzell für einen leidenden Klostervorsteher. Wiederholte ähnliche Anfragen treffen ein aus den Klöstern Einsiedeln, Neu St. Johann, Magdenau, Rorschach und aus Pfäfers über den dortigen Bädergebrauch. Aber selbst aus Lindau und Ulm wird Schobinger konsultiert; meist handelt es sich dabei um eingehende Berichte über Kranke und deren Zustände. Schobinger wird darin als höchste und letzte Instanz einer Hilfsmöglichkeit angerufen.

Wiederholt wendet sich der Straßburger Professor J. R. Salzmann (1653 bis 1656) an Schobinger. Salzmann war Professor der Medizin in Straßburg, Arzt und Dekan des Kapitels St. Thomas. Unter ihm wurden zwanzig Dissertationen angefertigt. Er schreibt $u$. a. über anatomische Beobachtungen an Patienten: «De anatomicis quibusdam observationibus». Vielfach wird er als Alchemist bezeichnet. Von ihm ist in der Vadiana seine Übersetzung von Thurneysens «Die 10 Bücher von kaltem und warmem Wasser» (1612) vorhanden. Salzmann schreibt an Schobinger über "Plantas quas misisti», ein andermal interpelliert er ihn über Magenstörungen oder über die Verwendung von Lobelium bei Epilepsie. Dann wieder holt er seinen Rat ein bei Krankheitsfällen, erörtert Fragen des Studiums usw. Eine sonderbare Persönlichkeit, die unter den Korrespondenten Schobingers wiederholt figuriert, lernen wir in Melchior Goldast kennen. Er stammt aus Espen bei Bischofszell, nennt sich Dr. jur., obwohl er nie eine Hochschule besuchte, und wird als Autodidakt bezeichnet, der Latein mit großer Gewandtheit beherrscht. Auch betätigt er ein abenteuerliches Wanderleben, das ihn weit herumführt, schreibt enorm viel, womit er sein Dasein fristet. Sein Tod erfolgte in Gießen. Mit Schobinger unterhält er sich über politische Fragen, so über den "Schmalkaldischen Bund». 
Bei J. C. Lavater, dem wir wiederholt in den Briefen an Schobinger begegnen, dürfte es sich um Johann Caspar Lavater (1586-1637) handeln, Professor der Physik am Karolinum in Zürich. Nicht nur der Zeit nach ist diese Annahme berechtigt, sondern auch der Inhalt der Briefe scheint es zu bestätigen. Lavater nennt Schobinger 1629 seinen "magne amice, vir clarissime et nobilissime» und seine "beneficia talia et tanta» dankt ihm für "beneficiis publice et privatim collatis», spricht von erhaltenen Bildern und Karten, von einem Planiglobium, wovon er die ebenfalls bei den Briefen erhaltene Skizze beilegt. Auch in einem späteren Briefe erwähnt er dieses Planiglobium.

Felix Platter (1536-1614), der Schobinger mit "prudentissime» anredet, berät ihn neben Familienangelegenheiten über medizinisch-therapeutische Fragen.

Von Thomas Platter (1614-28), Professor der Medizin und Botanik in Basel, dem jüngern Bruder von Felix, werden medizinische Probleme, ferner solche des Studiums und der Literatur aufgeworfen.

Theodor Zwinger (1597-1654), offenbar der Antistes, ein bekannter Basler Theologe, wendet sich 1626 an Schobinger als Bibliothekar der Vadiana, bespricht aber mit ihm auch aktuelle kirchliche Probleme, wie Fragen über die Eucharistie.

Auch von H.L.Peyer aus Schaffhausen sind einige Briefe erhalten. Es dürfte sich um den bischöflichen Rat, keinen Verwandten von J.C.Peyer (1653-1712), handeln. Es sind teils Weltanschauungsprobleme, so die Lehre Galileis, die ihn zu Schobinger führen. Aber auch über Fragen der Bibliothek, der Zeitpolitik, über persönliche und ärztliche Angelegenheiten erbittet er Schobingers Rat.

Wilhelm Fabricius Hildanus in Bern klagt über verlorene Briefe, berührt ebenfalls Bibliothekfragen und medizinische Probleme. Als Kuriosa und lebendige Zeugen von der außerordentlichen Verehrung, die Schobinger genoß, möge ein "Anagramme acrostiche d'honneur» auf Schobinger und seine Familie erwähnt sein, verfaßt und kalligraphisch ausgeführt von einer Onophria Zollikofer, ferner eine Huldigung in lateinischer Poesie eines Johann Christian Fabricius.

Unbekannt ist der Autor eines poetischen Lamentos in Form eines Akrostichons, betitelt «Der Königin Germaniae Klaglid». Es mutet derart zeitgemäß an, daß man es unsern Tagen entsprossen denken möchte. Darum sei es als Memento an das Elend nach dem Dreißigjährigen Krieg hier angefügt: 
Gering man mich achtet jetzund

Mich hatt man biss aufs Beyn verwund

Beraubet hatt man mir mein Gutt

Und gar ausgesogen mein Blutt

Man schind mir ab mein Haut und Haar

Mein Leben ist nichts mehr fürwar

Ich bin lebend todt und schabab

Jetzt trägt man mich lebend ins Grab

Niemand ist, der mir helfen thut

Jedermann dürstet nach meim Blut

Ich hab übersehen die Schanz

Und darumb kommen an diesen Dantz

An mir ein jeder spiegel sich

Und darumb lebe vorsichtiglich

$\mathrm{Da}$ ihn wie mich nicht greiff der Feind

Weil Practiquen obhanden seind.

Mag die hier bruchstückweise angedeutete Korrespondenz, auch wenn leider nur ihre zentripetale Komponente erhalten geblieben ist, uns von Ansehen und Bedeutung Schobingers untrügliches Zeugnis ablegen, so bleibt im Hinblick auf seine äußere Stellung die Frage ungeklärt: Wie kommt Schobinger, der Enkel eines führenden Vorkämpfers der Reformation, dazu, «fürstlich-sanktgallischer Stiftsarzt» zu werden? Schon über hundert Jahre vor ihm war 1457 im Berner Schiedsspruch vom 5. Februar die völlige Unabhängigkeit von Kloster und Stadt erklärt. Mit dem Jahr 1528 gilt die Reformation in der Stadt St. Gallen als abgeschlossen. Des fernern wissen wir, daß vor Schobinger zwei auswärtige Klosterärzte amteten, von 1522 bis 1535 Dr. Hans Rüssen aus Konstanz und Dr. Johannes Mürgel aus Lindau, beide also ohne ständigen Wohnsitz im Kloster. Offenbar wurden sie nach Bedarf zugezogen. Über Heilpflanzen und Einrichtungen für Schwitz- und Heilbäder verfügte das Kloster in reichem Maße (vgl. den von mir in unserer Gesellschaft früher beschriebenen Klosterplan). Schobinger wurde aber ausdrücklich zum "fürstlichen Stiftsarzt» ernannt, wohl nicht zuletzt vermöge seines hohen Ansehens als Arzt; doch gingen seine Beziehungen zum Kloster weit über seine ärztliche Tätigkeit hinaus. In anschaulicher Weise schildert das P. Mauritius Müller in den «Historiae Rerum S. Galli». Das Manuskript wurde mir in entgegenkom- 
mendster Weise von dem leider inzwischen verstorbenen Stiftsbibliothekar Dr. Müller zur Verfügung gestellt, die Übersetzung verdanke ich der sachverständigen Mithilfe von Herrn Dr. Gabathuler an der Vadiana. Schobinger wird dort als «lobenswerter, um den hl. Gallus hochverdienter Mann» dargestellt, den der damalige Abt Pius «wie seinen Vater verehrt und sich mit ihm häufig über religiöse Fragen unterhielt». Der Abt «hatte Schobinger häufig, ja fast täglich zu Gast und ihn wegen seiner außerordentlichen Geistesgaben mehr als alle andern geliebt». Es handelt sich um den Abt P. Pius Reher von Bleienried bei Weingarten (1597 bis 1654). Sein Klostereintritt erfolgte 1614. Er war Gründer der Stiftsdruckerei, Erbauer der Klosterkirche Neu St. Johann im Toggenburg, 18 Jahre jünger als Schobinger. Als dieser 73jährig starb, war der Abt 55, starb aber schon zwei Jahre später, ẹst 57jährig, "nach langer Krankheit, nachdem er im Bad Pfäfers die Heilung seines chronischen Leidens nicht gefunden hatte».

Die beiden Männer müssen sich also offenbar persönlich sehr nahegestanden haben, beide in der Antike geschult, philosophisch lebhaft interessiert, anscheinend in vielen Grundfragen übereinstimmend, was lag da näher, als daß der Jüngere den erheblich älteren Tischgenossen und Freund hoffte gänzlich für seine Auffassung gewinnen zu können. Das war der Grund, heißt es im zitierten Manuskript, «daß der Abt noch vertrauter sich mit ihm (Schobinger) unterhielt». Anläßlich eines gemeinsamen Tischgesprächs kommt die Rede auf «einen bevorstehenden Exorzismus bei zwei besessenen Nonnen, die der Abt gerade in seiner Obhut hatte, was Schobinger als «etwas Unmögliches verwarf», aber den Abt «um die Freundesgunst bat, ihm als Zuschauer Gelegenheit zu geben, seine fromme Neugier besser zu befriedigen». Das gewährt ihm der Abt bereitwilligst, allerdings nicht ohne die Mahnung, «falls vielleicht der Dämon eher unangenehme Dinge über ihn (Schobinger) ausschütte, solle er glauben, seine Neugier büßen zu müssen». Um diese Gefahr auszuschalten, erklärt Schobinger, von einem Winkel des Gotteshauses aus, durch eine verborgene Ritze einer Bretterwand, am Schauspiel teilzuhaben, «und findet sich dort ein, sehr früh am Morgen, bevor seine Person vom zusammenströmenden Volk bemerkt werden konnte». Während der Dämon schaurig tobte, rief er auch mit donnernder Stimme Schobinger an: «Das ist der Kerl, der weiß, daß er den wahren Glauben nicht hat, und sich trotzdem weigert, den falschen Glauben, dem er verschrieben ist, aufzugeben.» Wie sie nachher in üblicher Weise beim gemeinsamen Mahle 
sitzen, lacht Pius mit leichtem Spott Schobinger zu: «Bin ich etwa ein Prophet gewesen, Herr Bürgermeister?", worauf Schobinger antwortet: «Der Teufel ist verlogen, es kann und darf ihm also ein verständiger Mann nicht glauben.» - Warum ich diese Episode, auf die mich Kollege Milt hinzuweisen die dankenswerte Freundlichkeit hatte, hier erwähne? Sie scheint mir zum Charakterbild Schobingers zu gehören. Erst durch deren Kenntnis werden uns seine nahen Beziehungen zum Kloster eigentlich verständlich. Auf jeden Fall sind auch sie, wie seine Korrespondenz, sprechende Zeugen für Schobingers ärztliche und allgemeine Bedeutung in seiner Zeit.

\section{Neues vom St. Galler Schobinger-Bildnis des Paracelsus}

Von J.Strebel, Luzern, und D. Rittmeyer, St. Gallen

Sudhoff schreibt im zweiten Heft der Paracelsus-Forschungen (Schubert-Sudhoff, 1889, S. 141 ff., besonders S. 143) in bezug auf dieses heute in der Apotheke des Historischen Museums in St. Gallen deponierte Bildnis Hohenheims u. a. folgendes: «Schobingersche Familienpapiere werden heute noch auf der Stadtbibliothek St. Gallen aufbewahrt. Allein es war weder dort noch im Besitze der Familie selbst ein Brief oder ein Manuskript von Theophrastus aufzufinden. Auch anderweitige alchemistische Handschriften oder Briefschaften sollen nicht mehr vorhanden sein. Nur ein ölbildnis Hohenheims mit dunklem Vollbart und der Aufschrift ,Theophrastus Paracelsus 1529' soll aus Schobingerschem Familienbesitz in die Sammlung des Historischen Vereins in St. Gallen gekommen sein, wo es noch heute verwahrt wird. Dies Bild wäre also in St. Gallen der einzige dem Untergange entronnene Zeuge, daß einmal ein Glied dieser Familie Schobinger mit dem Arztgenius aus Einsiedeln in Beziehung gestanden hat."

Soweit die ersten Nachforschungen Sudhoffs, der S. 36 seines IX. Bandes sämtlicher Werke Hohenheims fortfährt: «Daß dieser St. Galler Bürger Schobinger (dessen Namen sein Zeitgenosse Rütiner, Lokalhistoriker, Schowinger schreibt) tatsächlich ein Porträt des Paracelsus besaß, ist dokumentarisch bewiesen. Ob aber das heute in St. Gallen verwahrte Bild mit diesem alten Schobingerschen Familienbesitz wirklich identisch ist, das ist mir recht zweifelhaft geworden. Das echte Bild war lange Jahrzehnte 